\title{
$q$-Convexity Properties of the Coverings of a Link Singularity
}

\author{
by \\ Mihnea ColţoiU, Cezar JoiţA and Mihai TiBĂR
}

\begin{abstract}
We prove that for a germ of normal isolated singularity $\left(Y, y_{0}\right)$ obtained by contracting a curve, if the fundamental group of the link singularity is infinite then the universal covering of $Y \backslash\left\{y_{0}\right\}$ can be written as the union of $n-1$ Stein open subsets.
\end{abstract}

2010 Mathematics Subject Classification: 32E10, 32F10, 32S45.

Keywords: q-complete space, Stein space, 1-convex manifold.

\section{§1. Introduction}

Let $\left(Y, y_{0}\right)$ be the germ of a normal 2-dimensional singularity and let $K$ be the associated link singularity. It was shown in [4] that if $\pi_{1}(K)$ is an infinite group then the universal covering of $Y \backslash\left\{y_{0}\right\}$ is Stein for $Y$ small enough.

In this paper we generalize this result to the case when $\left(Y, y_{0}\right)$ is a normal isolated singularity of dimension $n \geq 2$ obtained by contracting a complex curve. More precisely we prove:

Theorem. Suppose that $\left(Y, y_{0}\right)$ is a germ of normal isolated singularity obtained by contracting a curve, $\operatorname{dim}(Y)=n \geq 2$, and let $K$ be the corresponding link singularity. If $\pi_{1}(K)$ is infinite then the universal covering space of $Y \backslash y_{0}$ for $Y$ small can be written as the union of $n-1$ Stein open subsets. In particular it is $(n-1)$-complete.

Communicated by M. Kashiwara. Received December 10, 2010.

M. Colţoiu: Institute of Mathematics of the Romanian Academy, P.O. Box 1-764, Bucureşti 014700, Romania;

e-mail: Mihnea.Coltoiu@imar.ro

C. Joiţa: Institute of Mathematics of the Romanian Academy, P.O. Box 1-764,

Bucureşti 014700, Romania;

e-mail: Cezar. Joita@imar.ro

M. Tibăr: Mathématiques, UMR 8524 CNRS, Université des Sciences et Technologies de Lille, 59655 Villeneuve d'Ascq, France;

e-mail: tibar@math.univ-lille1.fr 
The theory of $q$-convexity was introduced by A. Andreotti and H. Grauert in [1] and is one of the basic tools in the study of the geometry of non-compact complex spaces.

\section{§2. Preliminaries}

For the following result see [15].

Theorem 1. Let $X$ be a complex space and $p: Y \rightarrow X$ a covering. If $X$ is Stein then $Y$ is Stein as well.

Theorem 2 was proved by Y. T. Siu in [14].

Theorem 2. If $X$ is a complex space and $Y$ is a Stein subspace then there exists an open Stein subset $U$ of $X$ such that $Y \subset U$.

Definition 1. Suppose that $X$ is a Stein space and $U$ is an open subset. We say that $U$ is Runge in $X$ (or that the pair $(U, X)$ is Runge) if $U$ is Stein and the restriction map $\mathcal{O}(X) \rightarrow \mathcal{O}(U)$ has dense image.

The following lemma is standard.

Lemma 1. Suppose that $X$ is a complex space and $\left\{X_{n}\right\}_{n \geq 1}$ is an increasing sequence of Stein open subsets of $X$. If each pair $\left(X_{n}, X_{n+1}\right)$ is Runge then $\bigcup_{n \geq 1} X_{n}$ is Stein.

For the next lemma see [10].

Lemma 2. Suppose that $X$ is a Stein space and $\phi: X \rightarrow \mathbb{R}$ is a plurisubharmonic function. Then for any $r \in \mathbb{R}$ the open set $U=\{x \in X: \phi(x)<r\}$ is Runge in $X$.

The lemma below follows from the fact that for a connected locally irreducible complex space the complement of a complex subspace of positive codimension is connected.

Lemma 3. Suppose that $\pi: X \rightarrow Y$ is a proper morphism of complex spaces and that there exists a discrete subset $A$ of $Y$ such that $\pi: X \backslash \pi^{-1}(A) \rightarrow Y \backslash A$ is a biholomorphism. If $X$ is locally irreducible then $Y$ is locally irreducible as well.

The following result is Theorem 2 in [13].

Theorem 3. Let $X$ be a locally irreducible Stein complex space of pure dimension 2 with isolated singularities and $A \subset X$ a closed complex subvariety without isolated points. Then $X \backslash A$ is Stein.

Remark. In [3] it was proved that if $\operatorname{dim}(X)=n \geq 2$ then $X \backslash A$ is the union of $n-1$ Stein open subsets. 
Using Theorem 3 and Lemma 3 we obtain:

Corollary 1. Let $X$ be a locally irreducible complex space of dimension 2 and $A \subset X$ a 1-dimensional closed complex subspace. Assume that $X$ is a proper modification of a Stein space at a discrete set of points, $A$ is connected, has at least one non-compact irreducible component, and $X \backslash A$ has no compact subspaces of positive dimension. Then $X \backslash A$ is Stein.

For the following proposition see [12, Remark a), p. 165].

Proposition 1. Suppose that $X$ is a 1-convex complex space and its exceptional set $A$ is 1-dimensional. Then $A$ has a neighborhood that can be embedded into a space $\mathbb{C}^{n} \times \mathbb{P}^{m}$.

The following theorem follows immediately from Theorem 2.4 in [12] using desingularization:

Theorem 4. Let $X$ be a 1-convex manifold which is embeddable into a space $\mathbb{C}^{n} \times \mathbb{P}^{m}$. Then there exist an open 1-convex neighborhood $V$ of the exceptional set and a complex projective manifold $Z$ such that $V$ is an open subset of $Z$.

The following theorem was proved in [2] and [9].

Theorem 5. Let $\pi: X \rightarrow T$ be a proper holomorphic surjective map of complex spaces, let $t_{0} \in T$ be any point, and denote by $X_{t_{0}}:=\pi^{-1}\left(t_{0}\right)$ the fiber of $\pi$ at $t_{0}$. Assume that $\operatorname{dim} X_{t_{0}}=1$. Let $\sigma: \tilde{X} \rightarrow X$ be a covering space and let $\tilde{X}_{t_{0}}=\sigma^{-1}\left(X_{t_{0}}\right)$. If $\tilde{X}_{t_{0}}$ is holomorphically convex, then there exists an open neighborhood $\Omega$ of $t_{0}$ such that $(\pi \circ \sigma)^{-1}(\Omega)$ is holomorphically convex.

Lemma 4. Suppose that $X$ is a Stein space and $U$ and $V$ are two Stein open subsets of $X$. If $(U, X)$ is Runge then $(U \cap V, V)$ is also Runge.

Proof. Let $K \subset U \cap V$ be a compact set. We have to show that there exists a plurisubharmonic function $\phi: V \rightarrow \mathbb{R}$ with $K \subset\{x \in V: \phi(x)<0\} \Subset U \cap V$. Let $\phi_{1}: X \rightarrow \mathbb{R}$ be a plurisubharmonic function such that $K \subset\left\{x \in X: \phi_{1}(x)<0\right\}$ $\Subset U$ and $\phi_{2}: V \rightarrow \mathbb{R}$ a plurisubharmonic exhaustion function such that $\phi_{2 \mid K}<0$. Then $\phi=\max \left\{\phi_{1}, \phi_{2}\right\}$ has the desired property.

Corollary 2. Suppose that $X$ is a complex space and $\Omega_{1}, \Omega_{2}, U_{1}$ and $U_{2}$ are open Stein subspaces of $X$ such that $U_{1}, U_{2} \subset \Omega_{1} \cap \Omega_{2}$. If $\left(U_{1}, \Omega_{1}\right)$ and $\left(U_{2}, \Omega_{2}\right)$ are Runge then $U_{1} \cap U_{2}$ is Runge in both $\Omega_{1}$ and $\Omega_{2}$.

The next lemma was proved in [4]. 
Lemma 5. Let $X$ be a Stein space and let $Y, U$ be Stein open subsets such that $X=U \cup Y$. Assume that $(Y \cap U, U)$ is Runge. Then $(Y, X)$ is also Runge.

Theorem 6 was proved in [5]; for a more general result see [6].

Theorem 6. Suppose that $X$ and $Y$ are complex analytic subsets of some neighborhood $U$ of the origin in $\mathbb{C}^{n}$ such that $0 \in Y, Y \subset X$ and $X \backslash Y$ is smooth. If the dimension of each component of $X \backslash Y$ is $\geq n$ and if $Y$ is defined in $X$ by $k$ holomorphic equations, then the pair $\left(X_{\epsilon} \backslash\{0\}, Y_{\epsilon} \backslash\{0\}\right)$ is $(n-k-1)$-connected for $\epsilon>0$ small enough.

In the above theorem $X_{\epsilon}=\{x \in X:\|x\| \leq \epsilon\}$ and similarly for $Y_{\epsilon}$. We also recall the following definition:

Definition 2. A pair $(X, A)$ with $A \stackrel{i}{\hookrightarrow} X$ is called $k$-connected if $i_{*}: \pi_{j}(A,\{a\})$ $\rightarrow \pi_{j}(X,\{a\})$ is bijective for $j<k$ and surjective for $j=k$, for all $a \in A$.

Corollary 3. Suppose that $X$ is a locally irreducible complex space such that all of its irreducible components have dimension at least $n$, and let $Y$ be a subspace of $X$. If $X \backslash Y$ is smooth and $Y$ is locally defined in $X$ by at most $n-2$ holomorphic equations, then $Y$ is locally irreducible.

We shall need the following:

Definition 3. Let $L$ be a connected 1-dimensional complex space and $\bigcup L_{i}$ be its decomposition into irreducible components. Then $L$ is called an infinite Nori string if all $L_{i}$ are compact and $L$ is not compact.

Definition 4. (a) If $\Omega$ is an open subset of $\mathbb{C}^{n}$, and $\psi: \Omega \rightarrow \mathbb{R}$ is a smooth function, then $\psi$ is called strictly $q$-convex if its Levi form has at least $n-q+1$ positive eigenvalues at every point.

(b) Suppose that $X$ is a complex space. A function $\phi: X \rightarrow \mathbb{R}$ is called strictly $q$-convex if for every $a \in X$ there exists an embedding of a neighborhood $U$ of $a$ as a closed analytic subset of an open subset $\Omega$ of $\mathbb{C}^{n}$, for some $n$, and a smooth strictly $q$-convex function $\psi: \Omega \rightarrow \mathbb{R}$ such that $\psi_{\mid U}=\phi$.

(c) A complex space $X$ is called $q$-complete if there exists a strictly $q$-convex exhaustion function $\phi: X \rightarrow \mathbb{R}$ (i.e. $\{x \in X: \phi(x)<c\} \Subset X$ for every $c \in \mathbb{R}$ ).

\section{$\S 3$. The results}

Proposition 2. Suppose that $Z$ is a complex projective variety with $\operatorname{dim}(Z)=n$ and $Y$ is a closed subvariety of $Z$ with $\operatorname{dim}(Y)=k$ such that $\operatorname{Sing}(Z) \subset \operatorname{Sing}(Y)$ 
and $k \leq(n-1) / 2$. Then there exists a principal hypersurface $H$ of $Z$ such that $Y \subset H$ and $\operatorname{Sing}(H) \subset \operatorname{Sing}(Y)$.

Proof. Let $L$ be a positive line bundle on $Z$ and let $\mathcal{I}$ the ideal of $Y$. It follows (see for example [8]) that there exists $m_{0} \in \mathbb{N}$ such that for any $m \geq m_{0}$ the canonical $\operatorname{map} \psi_{x}: \Gamma\left(Z, \mathcal{I} \otimes L^{m}\right) \rightarrow \Gamma\left(Z, \mathcal{I} / \mathfrak{m}_{x} \mathcal{I} \otimes L^{m}\right)$ is surjective for every $x \in Y$. If $x$ is a regular point of $Y$ then $\Gamma\left(X, \mathcal{I} / \mathfrak{m}_{x} \mathcal{I} \otimes L^{m}\right)=\Gamma\left(X, \mathcal{I} \otimes \mathcal{O} / \mathfrak{m}_{x} \otimes L^{m}\right)$ is a vector space of dimension $n-k$. It follows that for such a point $\operatorname{dim}\left(\operatorname{Ker}\left(\psi_{x}\right)\right)=N-n+k$ where $N=\operatorname{dim}\left(\Gamma\left(Z, \mathcal{I} \otimes L^{m}\right)\right)$.

We consider the diagram

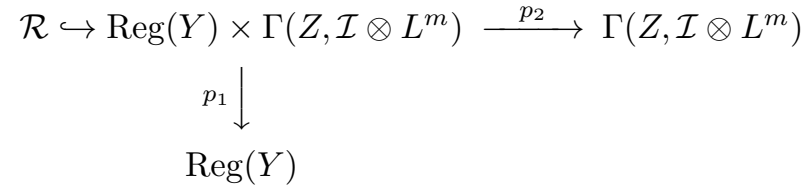

where $\mathcal{R}=\left\{(x, s) \in \operatorname{Reg}(Y) \times \Gamma\left(Z, \mathcal{I} \otimes L^{m}\right): \mathrm{d} s(x)=0\right\}$. Then $\operatorname{dim}\left(\mathcal{R} \cap p_{1}^{-1}(x)\right)=$ $N-n+k$ for every $x \in \operatorname{Reg}(Y)$ and hence $\operatorname{dim}(\mathcal{R}) \leq N-n+2 k$. We assumed that $k \leq(n-1) / 2$ and therefore $\operatorname{dim}(\mathcal{R})<N$. We deduce that $p_{2}(\mathcal{R})$ has measure zero. If $s$ is a section in $\Gamma\left(Z, \mathcal{I} \otimes L^{m}\right) \backslash p_{2}(\mathcal{R})$ and we set $H=\{x \in X: s(x)=0\}$ then $H$ is smooth at every point of $\operatorname{Reg}(Y)$. Also it follows from Bertini's theorem (see [7]) that for almost every $s$ the hypersurface $H$ is smooth at every point of $Z \backslash \operatorname{Sing}(Z)$, hence at every point of $Z \backslash Y$. We conclude that $\operatorname{Sing}(H) \subset \operatorname{Sing}(Y)$ for almost every $s \in \Gamma\left(Z, \mathcal{I} \otimes L^{m}\right)$.

Lemma 6. Suppose that $C$ is a 1-dimensional connected compact complex space such that $C$ has an irreducible component which is not locally irreducible. Then there exists a connected infinite Nori string $\tilde{C}$ and an unbranched covering map $p: \tilde{C} \rightarrow C$.

Proof. Let $C^{1}$ be an irreducible component of $C$ and $x_{0} \in C^{1}$ a point such that $C_{x_{0}}^{1}$, the germ of $C^{1}$ at $x_{0}$, is not irreducible. Let $\bigcup_{i \in I_{1}} C_{i, x_{0}}$ be the decomposition of $C_{x_{0}}^{1}$ into irreducible components (according to our assumption, $I_{1}$ has at least two elements), and $\bigcup_{i \in I} C_{i, x_{0}}$ the decomposition of $C_{x_{0}}$ into irreducible components with $I_{1} \subset I$. Let $U$ and $V$ be open neighborhoods of $x_{0}$ such that $\bar{U} \subset V$ and there exist closed analytic subsets $C_{i}, i \in I$, of $V$, which are representatives for $C_{i, x_{0}}$. We pick an index $j \in I_{1}$ and let $C^{\prime}=\bigcup_{i \in I \backslash\{j\}} C_{i}$, which is a closed analytic subset of $V$. Let $F:=\left((C \backslash \bar{U}) \sqcup C^{\prime} \sqcup C_{j}\right) / \sim$ where the equivalence relation is defined as follows. Let $x \in(C \backslash \bar{U}) \cap V$. Note that $(C \backslash \bar{U}) \cap V=\left(C^{\prime} \backslash \bar{U}\right) \cup\left(C_{j} \backslash \bar{U}\right)$ and that $C^{\prime} \backslash \bar{U}$ and $C_{j} \backslash \bar{U}$ are disjoint. Then if $x \in C^{\prime} \backslash \bar{U}$ we identify it with the corresponding point in $C^{\prime}$, while if $x \in C_{j} \backslash \bar{U}$ we identify it with the corresponding 
point in $C_{j}$. We have a projection $\tau: F \rightarrow C$ whose fiber above $x_{0}$ has exactly two elements, $P \in C^{\prime}$ and $Q \in C_{j}$, and $\tau: F \backslash\{P, Q\} \rightarrow C \backslash\left\{x_{0}\right\}$ is a biholomorphism. Note that $F$ is connected and compact.

Let now $\left\{F_{k}\right\}_{k \in \mathbb{Z}}$ be 1-dimensional complex spaces, each biholomorphic to $F$ via $\pi_{k}: F_{k} \rightarrow F$ and $P_{k}=\pi_{k}^{-1}(P), Q_{k}=\pi_{k}^{-1}(Q)$. We set $\tilde{C}=\left(\bigsqcup F_{k}\right) / \sim$ where $Q_{k}$ is identified with $P_{k-1}$. If we put $p: \tilde{C} \rightarrow C, p(x)=\tau\left(\pi_{k}(x)\right)$ for each $x \in F_{k} \backslash\left\{P_{k}, Q_{k}\right\}$ and $p\left(Q_{k}\right)=x_{0}$, we obtain an unramified covering. Obviously, $\tilde{C}$ is a connected infinite Nori string.

Remark. Let $T_{k}$ be the equivalence class of $F_{k}$ in $\tilde{C}$ and $z_{k}$ be the unique intersection point of $T_{k}$ and $T_{k+1}$ (i.e. $z_{k}$ is the equivalence class of $Q_{k}$ ). The identity map $F \rightarrow F$ induces a biholomorphism $T_{k} \rightarrow F$ which in turn induces a biholomorphism $g_{k}: T_{k} \rightarrow T_{k+1}$. Then $g: \tilde{C} \rightarrow \tilde{C}, g_{\mid T_{k}}=g_{k}$, is a (well-defined) covering transformation map.

Proposition 3. Let $X$ and $Y$ be two n-dimensional normal complex spaces, $n \geq 3, y_{0} \in Y$ and $\pi: X \rightarrow Y$ a proper holomorphic map such that $C=\pi^{-1}\left(y_{0}\right)$ is a connected 1-dimensional complex space and $\pi: X \backslash C \rightarrow Y \backslash\left\{y_{0}\right\}$ is a biholomorphism. Assume that $H_{1}(C)$ is infinite and that there exists a locally irreducible 2-dimensional complex subspace $S$ of $X$ with isolated singularities such that $C \subset S$. Then there exist an open neighborhood $W$ of $C$ in $X$ and an unbranched covering $p: \tilde{W} \rightarrow W$ such that $p^{-1}((S \backslash C) \cap W)$ is Stein.

Proof. We consider the decomposition $C=\bigcup C_{i}$ into irreducible components. Because $H_{1}(C)$ is infinite we distinguish three possible cases:

1. All irreducible components $C_{i}$ are locally irreducible, their graph is a (connected) tree, and at least one them has genus greater than or equal to 1 .

2. There exists an irreducible component $C_{i_{0}}$ which is not locally irreducible.

3. All irreducible components $C_{i}$ are locally irreducible, and their graph contains a cycle.

Case 1. In this case let $p: \tilde{C} \rightarrow C$ be a connected holomorphically convex covering of $C$ that has at least one non-compact irreducible component. There exists such a covering because at least one irreducible component of $C$ has genus greater than or equal to 1 . We also choose an open neighborhood $W_{1}$ of $C$ in $X$ such that $W_{1}$ has a continuous deformation retraction onto $W_{1} \cap S$ and $W_{1} \cap S$ has a continuous deformation retraction onto $C$. We extend the covering $p: \tilde{C} \rightarrow C$ to a covering $p: \tilde{W}_{1} \rightarrow W_{1}$, which in turn induces a covering $p: \tilde{S} \rightarrow S \cap W_{1}$. We apply Theorem 5 to deduce that we can find a neighborhood $W$ of $C$ in $X$ such that $p^{-1}(W)$ is holomorphically convex and therefore $p^{-1}(S \cap W)$ is holomorphically convex. Note 
that every compact 1-dimensional subspace of $p^{-1}(S \cap W)$ is included in $\tilde{C}$ and therefore $p^{-1}(S \cap W)$ is a proper modification of a Stein space at a discrete set of points. Corollary 1 then implies that $p^{-1}(S \cap W) \backslash \tilde{C}=p^{-1}((S \backslash C) \cap W)$ is Stein.

Case 2. We apply Lemma 6 to get a covering space $p: \tilde{C} \rightarrow C$ such that $\tilde{C}$ is an infinite Nori string. As in Case 1, we choose an open neighborhood $W_{1}$ of $C$ in $X$ such that $W_{1}$ has a continuous deformation retraction on $W_{1} \cap S$ and $W_{1} \cap S$ has a continuous deformation retraction in $S$ onto $C$, and we extend $p$ to a covering $p: \tilde{W}_{1} \rightarrow W_{1}$ which induces a covering $p: \tilde{S} \rightarrow S \cap W_{1}$. At the same time the covering transformation map $g$ extends to a covering transformation map $g: \tilde{S} \rightarrow \tilde{S}$. We are using here the notation of the proof of Lemma 6 and of the Remark that follows. Let $U_{0} \subset \tilde{S}$ be a strictly pseudoconvex, relatively compact neighborhood of $T_{0}$. For $k \in \mathbb{Z}, k>0$, we denote by $g^{(k)}$ the $k$-th iterate $g \circ \cdots \circ g$ and for $k \in \mathbb{Z}, k<0$, we put $g^{(k)}=\left(g^{-1}\right)^{(k)}$. We set $U_{k}=g^{(k)}\left(U_{0}\right)$. Then $U_{k}$ is a strictly pseudoconvex neighborhood of $T_{k}$. Shrinking $U_{0}$ we can assume that $\bar{U}_{0} \cap \bigcup_{|k| \geq 2} \bar{U}_{k}=\emptyset$ and that $p_{\mid U_{0} \cap U_{1}}$ and $p_{\mid U_{0} \cap U_{-1}}$ are 1-1. In particular $U_{0}$ does not contain any $T_{k}, k \neq 0$. It follows, obviously, that $U_{p} \cap U_{q}=\emptyset$ if $|k-p|>1$. By Corollary 1, $U_{k} \backslash T_{k-1}$ and $U_{k+1} \backslash T_{k+2}$ are Stein open subsets of $\tilde{S}$. We now choose an open Stein neighborhood $B_{0}$ of $z_{0}$ such that $B_{0} \subset U_{0} \cap U_{1}$ and $B_{0}$ is Runge in both $U_{0} \backslash T_{-1}$ and $U_{1} \backslash T_{2}$ (see Corollary 2). Moreover we assume that there exists an open Stein neighborhood $V_{1}$ of $y_{0}$ in $Y$ such that $V_{1} \supset p\left(\bar{B}_{0}\right)$. It follows from Lemma 4 that $B_{0} \backslash \tilde{C}$ is Runge in both $U_{0} \backslash \tilde{C}$ and $U_{1} \backslash \tilde{C}$. We set $B_{k}=g^{(k)}\left(B_{0}\right)$. Note that $p\left(B_{k}\right)=p\left(B_{0}\right)$ for every $k \in \mathbb{Z}$, and $B_{k} \backslash \tilde{C}$ is Runge in both $U_{k} \backslash \tilde{C}$ and $U_{k+1} \backslash \tilde{C}$.

We choose a strictly plurisubharmonic exhaustion function $\phi: V_{1} \rightarrow \mathbb{R}$ for $V_{1}$ such that $\phi\left(y_{0}\right)=0$ and $\phi(y)>0$ for $y \in V_{1} \backslash\left\{y_{0}\right\}$. Let $\epsilon>0$ be such that $V=\left\{y \in V_{1}: \phi(y)<\epsilon\right\} \Subset p\left(B_{0}\right)$. We claim that $(p \circ \pi)^{-1}(V \cap S) \backslash \tilde{C}$ is Stein. To prove this we consider for $k, l \in \mathbb{Z}, l<k, \Omega_{k, l}=\left(\bigcup_{j=l}^{k} U_{j}\right) \cap\left((p \circ \pi)^{-1}(V \cap S)\right)$ and $M_{k, l}=\Omega_{k, l} \backslash \tilde{C}$. Note that since $p\left(\partial U_{j_{1}} \cap \partial U_{j_{2}}\right) \cap B_{0}=\emptyset$ for $j_{1} \neq j_{2}$, each $\Omega_{k, l}$ is a strictly pseudoconvex, relatively compact open subset of $\tilde{S}$. Its maximal compact 1-dimensional subvariety is $T_{l} \cup \cdots \cup T_{k}$, which is exceptional. Hence $\Omega_{k, l}$ is 1-convex. On the other hand $\tilde{C} \cap \Omega_{k, l}=\left(\bigcup_{j=l}^{k} T_{j}\right) \cup\left(T_{k+1} \cap \Omega_{k, l}\right) \cup\left(T_{l-1} \cap \Omega_{k, l}\right)$. Because $\Omega_{k}$ does not contain $T_{k+1}$ or $T_{l-1}$ it follows from Corollary 1 that $M_{k, l}$ is Stein. Note now that $M_{k+1, l}=M_{k, l} \cup\left(\left(U_{k+1} \cap(p \circ \pi)^{-1}(V \cap S)\right) \backslash \tilde{C}\right)$ and that $M_{k, l} \cap\left(\left(U_{k+1} \cap(p \circ \pi)^{-1}(V \cap S)\right) \backslash \tilde{C}\right)=\left(B_{k} \cap(p \circ \pi)^{-1}(V \cap S)\right) \backslash \tilde{C}$, which by Lemma 2 is Runge in $\left(U_{k+1} \cap(p \circ \pi)^{-1}(V \cap S)\right) \backslash \tilde{C}$. We deduce from Lemma 5 that $M_{k, l}$ is Runge in $M_{k+1, l}$. Similarly $M_{k, l}$ is Runge in $M_{k, l-1}$. Therefore $M_{k,-k}$ is Runge in $M_{k+1,-k-1}$ for every $k \in \mathbb{Z}, k>0$. As $(p \circ \pi)^{-1}(V \cap S) \backslash \tilde{C}=\bigcup_{k=1}^{\infty} M_{k+1,-k-1}$ it follows from Lemma 1 that $(p \circ \pi)^{-1}(V \cap S) \backslash \tilde{C}$ is Stein as claimed. 
Case 3. Let $C_{1}, \ldots, C_{k}$ be irreducible components of $C$ such that their graph forms a minimal cycle (i.e. no proper subset of $\left\{C_{1}, \ldots, C_{k}\right\}$ forms a cycle). We contract $C_{2} \cup \cdots \cup C_{k}$ in $X$ to obtain a normal complex space $X^{\prime}$. Let $S^{\prime}$ and $C^{\prime}$ be the images of $S$ and $C$ respectively. It follows from Lemma 3 that $S^{\prime}$ is locally irreducible. Notice at the same time that $C^{\prime}$ is not locally irreducible anymore and hence we can apply Case 2. We obtain a neighborhood $W^{\prime}$ of $C^{\prime}$ and a covering map $p^{\prime}: \tilde{W}^{\prime} \rightarrow W^{\prime}$ such that $p^{-1}\left(\left(S^{\prime} \backslash C^{\prime}\right) \cap W^{\prime}\right)$ is Stein. We pull back this covering via the contraction map to obtain a covering for a neighborhood of $C$ with the desired property.

Theorem 7. Suppose that $\left(Y, y_{0}\right)$ is a germ of normal isolated singularity obtained by contracting a curve, $\operatorname{dim}(Y)=n \geq 2$, and let $K$ be the corresponding link singularity. If $\pi_{1}(K)$ is infinite, then the universal covering space of $Y \backslash y_{0}$ for $Y$ small can be written as the union of $n-1$ Stein open subsets. In particular it is $(n-1)$-complete.

Proof. If $\operatorname{dim}(Y)=2$ the theorem was proved in [4]. Hence we assume that $\operatorname{dim}(Y) \geq 3$. Let $\pi: X \rightarrow Y$ be a local resolution of singularities and $C$ the exceptional curve. As we are assuming that $n \geq 3$ it follows that $H_{1}(C)$ is infinite since $\pi_{1}(C)$ is infinite (note that $C$ has real codimension $>2$ in $X$ so $\pi_{1}(X)=\pi_{1}(X \backslash C)$ ). On the other hand from Proposition 1 it follows that $C$ has a strictly pseudoconvex neighborhood which can be embedded into a space $\mathbb{C}^{n} \times \mathbb{P}^{m}$, and then by Theorem 4, there exist an open 1-convex neighborhood $V$ of the exceptional set and a complex projective manifold $Z$ such that $V$ is an open subset of $Z$. We will show now that we can find a two-dimensional locally irreducible subvariety $S$ of $Z$ such that $\operatorname{Sing}(S) \subset \operatorname{Sing}(C)$ and $Z \backslash S$ is the union of $n-2$ Stein open subsets. The local irreducibility will follow from Corollary 3 if we can choose $S$ to be a local set-theoretic complete intersection. To obtain $S$ we apply Proposition $2, n-2$ times, to we obtain a sequence of projective varieties $H_{1} \supset \cdots \supset H_{n-2}=: S \supset C$ such that $H_{j+1}$ is a principal hypersurface in $H_{j}$ and $\operatorname{Sing}\left(H_{j}\right) \subset \operatorname{Sing}(C)$. Each $H_{j} \backslash H_{j+1}, j=1, \ldots, n-3$, is Stein and Theorem 2 implies that there exists a Stein open subset $\Omega_{j+1}$ of $Z$ such that $\Omega_{j+1} \cap H_{j}=H_{j} \backslash H_{j+1}$. If we put $\Omega_{1}=Z \backslash H_{1}$ we get $Z \backslash H_{n-2}=Z \backslash S=\Omega_{1} \cup \cdots \cup \Omega_{n-2}$. In particular, since $V$ is strictly pseudoconvex, $V \backslash S$ is the union of $n-2$ Stein open subsets.

We now apply Proposition 3 to find a strictly pseudoconvex neighborhood $W$ of $C$ in $X$ such that on one hand $W \backslash S=W_{1} \cup \cdots \cup W_{n-2}$ where $W_{j}, j=1, \ldots, n-2$, are Stein open subsets of $X$ and on the other hand there exists an (unbranched) covering space $p: \tilde{W} \rightarrow W$ for which $p^{-1}((S \backslash C) \cap W)$ is Stein.

It remains to notice is that $\tilde{W}_{j}:=p^{-1}\left(W_{j}\right), j=1, \ldots, n-2$, are Stein (see Theorem 1) and, at the same time, by Theorem 2 there exists a Stein open 
subset $\tilde{W}_{n-1}$ of $\tilde{W}$ such that $\tilde{W}_{n-1} \cap p^{-1}(S)=p^{-1}((S \backslash C) \cap W)$. Obviously $\tilde{W}=\tilde{W}_{1} \cup \cdots \cup \tilde{W}_{n-1}$ and hence $\tilde{W}$ is the union of $n-1$ Stein open sets. As the universal covering $\hat{W}$ of $W$ is a covering of $\tilde{W}$, Theorem 1 implies that $\hat{W}$ is the union of $n-1$ Stein open sets. The $(n-1)$-completeness of $\hat{W}$ follows from $[11$, Satz 2.3].

\section{Acknowledgements}

The first two authors were partially supported by CNCSIS Grant PN-II ID_1186, contract $455 / 2008$.

\section{References}

[1] A. Andreotti and H. Grauert, Théorème de finitude pour la cohomologie des espaces complexes, Bull. Soc. Math. France 90 (1962), 193-259. Zbl 0154.33601 MR 0150342

[2] M. Colţoiu and K. Diederich, On the coverings of proper families of 1-dimensional complex spaces, Michigan Math. J. 47 (2000), 369-375. Zbl 1001.32007 MR 1793631

[3] Convexity properties of analytic complements in Stein spaces, in Proceedings of the conference in honor of Jean-Pierre Kahane (Orsay, 1993), J. Fourier Anal. Appl. 1995, Special Issue, 153-160. Zbl 1001.32007 MR 1793631

[4] M. Colţoiu and M. Tibăr, Steinness of the universal covering of the complement of a 2-dimensional complex singularity, Math. Ann. 326 (2003), 95-104. Zbl 1039.32038 MR 1981613

[5] H. Hamm, Lokale topologische Eigenschaften komplexer Räume, Math. Ann. 191 (1971), 235-252. Zbl 0214.22801 MR 0286143

[6] , On the vanishing of local homotopy groups for isolated singularities of complex spaces, J. Reine Angew. Math. 323 (1981), 172-176. Zbl 0483.32007 MR 0611450

[7] J. Harris, Algebraic geometry. A first course, Grad. Texts in Math. 133, Springer, New York, 1995. Zbl 0779.14001 MR 1416564

[8] R. Hartshorne, Algebraic geometry, Grad. Texts in Math. 52, Springer, New York, 1977. Zbl 0367.14001 MR 0463157

[9] T. Napier, Convexity properties of coverings of smooth projective varieties, Math. Ann. 28 (1990), 433-479. Zbl 0733.32008 MR 1032941

[10] R. Narasimhan, The Levi problem for complex spaces. II, Math. Ann. 146 (1962), 195-216. Zbl 0131.30801 MR 0182747

[11] M. Peternell, Algebraische Varietäten und $q$-vollständige komplexe Räume, Math. Z. 200 (1989), 547-581. Zbl 0675.32014 MR 0987586

[12] T. Peternell, On strongly pseudoconvex Kähler manifolds, Invent. Math. 70 (1982/83), 157-168. Zbl 0505.32023 MR 0684170

[13] R. R. Simha, On the complement of a curve on a Stein space of dimension two, Math. Z. 82 (1963), 63-66. Zbl 0112.31103 MR 0153869

[14] Y. T. Siu, Every Stein subvariety admits a Stein neighborhood, Invent. Math. 38 (1976/77), 89-100. Zbl 0343.32014 MR 0435447

[15] K. Stein, Überlagerungen holomorph-vollständiger komplexer Räume, Arch. Math. (Basel) 7 (1956), 354-361. Zbl 0072.08002 MR 0084836 